\title{
A DIRECT APPROACH FOR THE LINDELÖF CONJECTURE RELATED TO THEORY OF THE RIEMANN ZETA FUNCTION
}

\author{
XIAO-JUN YANG ${ }^{1,2,3}$
}

\begin{abstract}
It is due to Littlewood that the truth of the Riemann theorem implies that of the Lindelöf conjecture. This paper aims to use the idea of Littlewood to prove the Lindelöf conjecture for the Riemann zeta function. The Lindelöf $\mu$ function at the critical line is zero, with use of the Riemann theorem for the entire Riemann zeta function, proved based on the work of Heath-Brown. Our result is given to show that the Lindelöf conjecture, connected with the proof of the moment conjecture, is true.
\end{abstract}

\section{COnTEnts}

1. Introduction 1

2. Preliminaries 4

3. The Lindelöf conjecture is true 8

3.1. The new proof of the Riemann theorem 9

3.2. The proof of the Lindelöf conjecture 12

4. The moment conjecture is true 13

5. New results on the moment for the Riemann zeta function 14

$\begin{array}{ll}\text { References } & 18\end{array}$

\section{InTRODUCTION}

The Lindelöf conjecture via Riemann zeta function, proposed in 1908 by Finnish mathematician Ernst Leonard Lindelöf, has been one of most important open problems in the history of mathematics [1]. More important, the Lindelöf conjecture is not only linked with the consequence of the Riemann conjecture [2] but also used to investigate the higher movement for the Riemann zeta function [3]. The Lindelöf conjecture has played the important role in the field of the analytic number theory [4].

1991 Mathematics Subject Classification. 11M06, 11M26.

Key words and phrases. Lindelöf conjecture, Riemann zeta function, nontrivial zeros, Riemann theorem, moment conjecture. 
Suppose that $\mathcal{C}, \mathcal{R}$ and $\mathcal{N}$ are the sets of the complex numbers, real numbers and natural numbers, respectively. Let $s=\sigma+i t \in \mathcal{C}$ such that $\operatorname{Re}(s)=\sigma \in \mathcal{R}$ and $\operatorname{Im}(s)=t \in \mathcal{R}$ are the real and imaginary parts of the complex variable $s$, where $i=\sqrt{-1}$. Let As is well known, the Riemann zeta function $\zeta(s)$ of the complex variable $s=\sigma+i t$ is defined by the sum [5]

$$
\zeta(s)=\sum_{k=1}^{\infty} k^{-s}
$$

where $k \in \mathcal{N}$ and $R e(s)>1$. As is stated in [5] that this allows Eq. (1) to be a meromorphic continuation to the entire complex plane $s$, with pole of residue 1 at $s=1$. The trivial zeros for Eq. (1) reads $s=-2 \nu$ with $\nu \in \mathcal{N}$. The nontrivial zeros for Eq. (1) are located on the critical line $R e(s)=1 / 2$ and in the critical trip $0<\operatorname{Re}(s)<1[6,7]$. The entire Riemann zeta function $\xi(s)$ is expressed by the product of the Riemann zeta function $\zeta(s)$ or the series $[5,8]$ :

$$
\xi(s)=\zeta(s) \Pi(s)=\sum_{h=1}^{\infty} \vartheta(h)\left(s-\frac{1}{2}\right)^{2 h},
$$

where $\Gamma$ is the gamma function [9],

$$
\Pi(s)=(s-1) \pi^{-s / 2} \Gamma(s / 2+1)
$$

and [8]

$$
\vartheta(h)=\frac{4}{(2 h) !} \int_{1}^{\infty} \frac{d\left(z^{\frac{3}{2}} \psi^{(1)}(x)\right)}{d x} x^{-\frac{1}{4}}\left(\frac{\log x}{2}\right)^{2 h} d x
$$

with $h \in \mathcal{R} \cup\{0\}$ and $\psi(x)=\sum_{v=1}^{\infty} e^{-v^{2} \pi x}$.

Based on the above-mentioned results, it is stated in 2005 by Heath-Brown [7] that an equivalent statement for the Riemann theorem [5] is given as follows:

Theorem 1. Riemann theorem (Heath-Brown statement) The Heath-Brown statem states the real part of all zeros of $\xi(s)$ is $1 / 2$.

It is equivalent to the Riemann statement [5] that the real part of the nontrivial zeros of $\zeta(s)$ is $1 / 2$. It is known that Eq. (2) is the entire function of order 1 [10]. Although the Riemann statement has been achieved in [11], we would like to give the proof of the Heath-Brown statement for the Riemann theorem. For more details for the zeros, number of zeros and imaginary parts of zeros for $\xi(s)$, see $[8,10]$.

Based on the above, the Lindelöf conjecture [1] claims that for every positive $\varepsilon>0$,

$$
\zeta(\sigma+i t) \ll t^{\varepsilon}
$$


where $t \rightarrow \infty$.

This easily yields that the equivalent statement for the Lindelöf conjecture as follows $[8,10,12]$ :

\section{Conjecture 1. Lindelöf conjecture}

There exists

$$
\zeta\left(\frac{1}{2}+i t\right) \ll t^{\varepsilon}
$$

for every positive $\varepsilon>0$, and $t \rightarrow \infty$.

There are a number of the equivalent statements for the Lindelöf conjecture. The equivalences of these various assertions were proposed in 1915 by Hardy and Riesz [12] and reported in 2015 by Conrey [13]. It is shown in 1912 by Littlewood that Conjecture 1 is the consequences of Theorem 1 implies [2]. In 1923, Hardy and Littlewood give two equivalences for Conjecture 1 states that [3]

$$
\frac{1}{T} \int_{1}^{T}\left|\zeta\left(\frac{1}{2}+i t\right)\right|^{2 m} d t \ll T^{\varepsilon}
$$

for $\varepsilon>0, m \in \mathcal{N}$ and $T \rightarrow \infty$, and

$$
\frac{1}{T} \int_{1}^{T}|\zeta(\sigma+i t)|^{2 m} d t \ll T^{\varepsilon}
$$

for $\varepsilon>0, m \in \mathcal{N}, \sigma \geq \frac{1}{2}$ and $T \rightarrow \infty$.

It is stated in 2006 by Laurincikas and Steuding that the equivalence for the Lindelöf conjecture becomes [14]

$$
\int_{0}^{T}\left|\zeta\left(\frac{1}{2}+i t\right)\right|^{2 m} d t \ll T^{\varepsilon+1}
$$

for $\varepsilon>0, m \in \mathcal{N}$ and $T \rightarrow \infty$.

Eq. (9) implies that the moment conjecture states [15]

$$
\frac{1}{T} \int_{0}^{T}\left|\zeta\left(\frac{1}{2}+i t\right)\right|^{2 m} d t \ll T^{\varepsilon},
$$

for $\varepsilon>0, m \in \mathcal{N}$ and $T \rightarrow \infty$.

There exist the advances for the Lindelöf conjecture, reported in 2006 by Conrey and Ghosh [16] and made in 2019 by Fokas [17] based on the estimation of relevant exponential sums. 
Note that $\mu(\sigma)$ is the Lindelöf $\mu$ function, expressed in Eq. (5) [1]. It was proved that (see [2, 18]; [12],p.18)

$$
\mu(\sigma)=\left\{\begin{array}{l}
0, \text { for } \sigma \geq 1 / 2 \\
1 / 2-\sigma, \text { for } \sigma \leq 1 / 2
\end{array}\right.
$$

Due to the idea of Littlewood [2], which is the only way of proving the Lindelöf conjecture, the target of the paper is to give the proof of the Lindelöf conjecture by the study of the Lindelöf $\mu$ function (see [12], p.18; [18], p.338), with the aid of Theorem 1, which is proved based on the work of Heath-Brown [7]. The structure of the paper is given as follows. In Section 2 we introduce the results for the Riemann zeta function. In Section 3 we present the proof of Conjecture 1. In Section 4 we prove the moment conjecture. Finally, we suggest the new results on the moment for the Riemann zeta function in Section 5.

\section{Preliminaries}

In this section we give the recent results on the Riemann $\Xi$ and entire Riemann zeta functions.

Let $s_{n}, \varphi_{n}$ and $\phi_{n}$ run the nontrivial zeros of the Riemann zeta function $\zeta(s)$, the imaginary part of the Riemann zeta function $\zeta(s)$, and the positive imaginary part of the Riemann zeta function $\zeta(s)$, respectively.

Lemma 1. Let $s \in \mathcal{C}$ and $\wp=\log 2+\frac{1}{2} \log \pi-1-\frac{1}{2} \varpi$, where $\varpi$ is the Euler's constant. Suppose that $\widetilde{s_{n}}=\operatorname{Re}\left(s_{n}\right)+\phi_{n}$, then the following representations are equivalent:

$$
\begin{gathered}
\xi(s)=\xi(0) \prod_{n=1}^{\infty}\left(1-\frac{s}{s_{n}}\right) \\
\xi(s)=\xi(1 / 2) \prod_{n=1}^{\infty}\left(1+\frac{i(s-1 / 2)}{\varphi_{n}}\right), \\
\xi(s)=\xi(0) e^{s \wp} \prod_{n=1}^{\infty}\left(1-\frac{s}{s_{n}}\right) e^{s / s_{n}}, \\
\xi(s)=\xi(1 / 2) e^{s \wp} \prod_{l=1}^{\infty}\left(1+\frac{i(s-1 / 2)}{\varphi_{n}}\right) e^{s /\left(1 / 2+i \varphi_{n}\right)} \\
\xi(s)=\xi(1 / 2) \prod_{n=1}^{\infty}\left(1-\frac{(s-1 / 2)^{2}}{\phi_{n}^{2}}\right)
\end{gathered}
$$




$$
\begin{gathered}
\xi(s)=\xi\left(\frac{1}{2}\right) \prod_{n=1}^{\infty}\left[1-\frac{\left(s-\frac{1}{2}\right)^{2}}{\left(\widetilde{s_{n}}-\frac{1}{2}\right)^{2}}\right], \\
\xi(s)=\xi(0) \prod_{n=1}^{\infty}\left(1-\frac{s}{\widetilde{s_{n}}}\right)\left(1-\frac{s}{1-\widetilde{s_{n}}}\right), \\
\xi(s)=\xi(0) \prod_{n=1}^{\infty}\left[1-\frac{s(1-s)}{\widetilde{s_{n}}\left(1-\widetilde{s_{n}}\right)}\right],
\end{gathered}
$$

and

$$
\xi(s)=\sum_{h=1}^{\infty} \vartheta(h)\left(s-\frac{1}{2}\right)^{2 h}
$$

where

$$
\vartheta(h)=\frac{4}{(2 h) !} \int_{1}^{\infty} \frac{d\left(z^{\frac{3}{2}} \psi^{(1)}(x)\right)}{d x} x^{-\frac{1}{4}}\left(\frac{\log x}{2}\right)^{2 h} d x .
$$

Proof. See the details for the proof of Lemma 1 [11].

Remark. The Hadamard product (12) was discovered by Hadamard in 1893 [19]. Eq. (13) was discovered by Edwards [8] and proved by author in three ways [11]. Eq. (14) was discovered by Hadamard in 1893 [19], discussed by Landau in 1909 [20] and by Titchmarsh in 1930 [21], and proved in 1964 by Ingham [4]. Eq. (15), discovered in [11], was derived from Eq. (14). Eq. (16) was obtained by Eq. (17) based on the Riemann theorem [11]. Eq. (98), derived from the Patterson product (see [22], p.34), e.g.,

$$
2 \zeta(s) \Pi(s)=\prod_{n=1}^{\infty}\left(1-\frac{s}{\widetilde{s_{n}}}\right)\left(1-\frac{s}{1-\widetilde{s_{n}}}\right),
$$

where $s \in \mathcal{C}$, leads to the equivalences of Eqs. (98) and (99) by author [11]. Both (98) and (99) can be connected, as shown by Edwards in 1974 [8]. Eq. (100) was discovered by Edwards in 1974 [8]. From Eq. (14) we see that $\xi(s)$ is the entire function of order $1[7]$.

Lemma 2. (Turán inequalities [11, 13, 23, 24])

Let $h \geq 0$. Then the Turán inequalities

$$
(\vartheta(h))^{2}-\left(\frac{2 h-1}{2 h+1}\right) \vartheta(h-1) \vartheta(h+1)>0
$$

hold for any $h \in \mathcal{N} \cup\{0\}$. 
Proof. See the proof of Lemma 2 [11, 13, 23, 24].

Lemma 3. (Hardy theorem [10, 25])

The Hardy theorem states that the entire Riemann zeta function $\xi(s)$ has infinitely many zeros.

Proof. For the details of the proof of Lemma 3, see [10, 25].

Lemma 4. The entire Riemann zeta function $\xi(s)$ has infinitely many zeros $s_{n} \in \mathcal{C}$.

Proof. For the proof of Lemma 4, see [11].

Remark. Lemma 4 can be derived from Lemmas 2 and 3.

Let $s=\frac{1}{2}+i t$ such that $[20]$

$$
\xi\left(\frac{1}{2}+i t\right)=\Xi(t),
$$

where $t \in \mathcal{C}$.

Lemma 5. Let $s \in \mathcal{C}$ and $\wp=\log 2+\frac{1}{2} \log \pi-1-\frac{1}{2} \varpi$, where $\varpi$ is the Euler's constant. Suppose that $\widetilde{s_{n}}=\operatorname{Re}\left(s_{n}\right)+\phi_{n}$, then the following representations are equivalent:

$$
\begin{gathered}
\Xi(t)=\xi(0) \prod_{n=1}^{\infty}\left(1-\frac{\frac{1}{2}+i t}{s_{n}}\right), \\
\Xi(t)=\xi(1 / 2) \prod_{n=1}^{\infty}\left(1-\frac{t}{\varphi_{n}}\right), \\
\Xi(t)=\xi(0) e^{\wp\left(\frac{1}{2}+i t\right)} \prod_{n=1}^{\infty}\left(1-\frac{\frac{1}{2}+i t}{s_{n}}\right) e^{(1 / 2+i t) / s_{n}}, \\
\Xi(t)=\xi(1 / 2) e^{\wp\left(\frac{1}{2}+i t\right)} \prod_{l=1}^{\infty}\left(1-\frac{t}{\varphi_{n}}\right) e^{(1 / 2+i t) /\left(1 / 2+i \varphi_{n}\right)}, \\
\Xi(t)=\xi(1 / 2) \prod_{n=1}^{\infty}\left(1-\frac{t^{2}}{\phi_{n}^{2}}\right), \\
\Xi(t)=\xi\left(\frac{1}{2}\right) \prod_{n=1}^{\infty}\left[1+\frac{t^{2}}{\left(\widetilde{s_{n}}-\frac{1}{2}\right)^{2}}\right]
\end{gathered}
$$




$$
\begin{gathered}
\Xi(t)=\xi(0) \prod_{n=1}^{\infty}\left(1-\frac{\frac{1}{2}+i t}{\widetilde{s_{n}}}\right)\left(1-\frac{\frac{1}{2}+i t}{1-\widetilde{s_{n}}}\right), \\
\Xi(t)=\xi(0) \prod_{n=1}^{\infty}\left[1-\frac{\frac{1}{4}+t^{2}}{\widetilde{s_{n}}\left(1-\widetilde{s_{n}}\right)}\right],
\end{gathered}
$$

and

$$
\Xi(t)=\sum_{h=1}^{\infty} \vartheta(h)(-1)^{h} t^{2 h}
$$

where

$$
\vartheta(h)=\frac{4}{(2 h) !} \int_{1}^{\infty} \frac{d\left(z^{\frac{3}{2}} \psi^{(1)}(x)\right)}{d x} x^{-\frac{1}{4}}\left(\frac{\log x}{2}\right)^{2 h} d x .
$$

Proof. For the details for the proof of Lemma 5, see [11].

Lemma 6. The Riemann $\Xi$ function $\Xi(t)$ with $t \in \mathcal{C}$ has infinitely many real zeros $\varphi_{n} \in \mathcal{R}$.

Proof. For the proof of Lemma 6, see [11].

Remark. It is well known that if $\vartheta(h)$ is given, then

$$
\sum_{h=1}^{\infty} \vartheta(h)(-1)^{h} t^{2 h}=\xi(1 / 2) e^{\hbar_{0}\left(\frac{1}{2}+i t\right)} \prod_{l=1}^{\infty}\left(1-\frac{t}{\varphi_{n}}\right) e^{(1 / 2+i t) /\left(1 / 2+i \varphi_{n}\right)}
$$

is in the Laguerre-Pólya class [26, 27].

Eq. (24) was derived from the Hadamard product by author [11]. Eq. (25), discovered in [11], was derived from Eq. (24) based on the Riemann theorem. As shown in [11], Eq. (26) was derived from the Hadamard product (14) when one takes $s=1 / 2+i t$ in Eq. (14). Eq. (27), discovered in [11], was derived from Eq. (26) with the aid of Eqs. (24) and (25). Eq. (28), reported in 1894 by Cahen [28] and further discussed in 1927 by Titchmarsh [29], was deduced by the formula (here take the principal value)

$$
\log \Xi(t)=\log \xi(1 / 2)+\sum_{n=1}^{\infty}\left(1-\frac{t^{2}}{\phi_{n}^{2}}\right),
$$

which implies that [5]

$$
\log \Xi(t)=\log \xi(1 / 2)+\sum_{n=1}^{\infty}\left(1-\frac{t^{2}}{\phi_{n}^{2}}\right),
$$


where

$$
\xi(1 / 2)=\Xi(0)>0 .
$$

Eq. (28) is in the case of Eq. (29) when the Riemann theorem is valid. Eq. (29) was derived by Eq. (17), which was deduced by the Patterson product [22]. Eqs. (30) and (31), discovered in [11], were equivalent to Eq. (29) since Lemma 1 is valid for $s=1 / 2+i t$. Eq. (32) was proposed by Hadamard [19], further studied by Jensen in 1913 [30], and discussed by Pólya and Schur in 1914 [27] and by Pólya in 1927 [31]. It is easily seen that $\Xi(t)$ is the even entire function of order 1 [10], and that both $\varphi_{n}$ and $\phi_{n}$ are imaginary parts, obtained by the Riemann-Siegel formula [32]. Here, the imaginary parts $\varphi_{n}$ and $\phi_{n}$ are called as the Riemann-Siegel zeros.

Lemma 7. (Montgomery and Vaughan [18])

Let $\aleph>0$ be fixed. Then

$$
\Im_{1} t^{\frac{1}{2}-\sigma}|\zeta(1-s)| \leq|\zeta(s)| \leq \Im_{2} t^{\frac{1}{2}-\sigma}|\zeta(1-s)|
$$

uniformly for $|\sigma| \leq \aleph$, some positive absolute constants $\Im_{1}>0$ and $\Im_{2}>0$, and $|t| \geq 1$.

Proof. See the result of Montgomery and Vaughan (see [18], p.330).

Lemma 8. Suppose that $\mu(\sigma)$ is the Lindelöf $\mu$ function. Then,

$$
\mu(\sigma)=\left\{\begin{array}{l}
0, \text { for } \sigma \geq 1 / 2, \\
1 / 2-\sigma, \text { for } \sigma \leq 1 / 2 .
\end{array}\right.
$$

Proof. According to Hardy and Riesz (see [12], p.18) and Montgomery and Vaughan (see [18], p.338), we set

$$
\Re(T)=\max _{0 \leq t \leq T}|\zeta(1 / 2+i t)|
$$

If $\Re(T) \ll T^{\varepsilon}$, then (see [18], p.338)

$$
\mu(\sigma)=0
$$

for $\sigma \geq 1 / 2$.

By Lemma 7 it is shown that if $\Re(T) \ll T^{\varepsilon}$, then (see [18], p.338)

$$
\mu(\sigma)=\frac{1}{2}-\sigma
$$

for $\sigma \leq 1 / 2$.

\section{The LindeLÖF CONJECTURE IS TRUE}

In this section we prove the Lindelöf conjecture by using the Heath-Brown statement of the Riemann theorem. 
3.1. The new proof of the Riemann theorem. We now consider the new proof of Theorem 1.

From the Hadamard product [19]

$$
\xi(s)=\xi(0) \prod_{n=1}^{\infty}\left(1-\frac{s}{s_{n}}\right),
$$

and the Patterson product (see [22], p.34)

$$
2 \xi(s)=\prod_{n=1}^{\infty}\left(1-\frac{s}{\widetilde{s_{n}}}\right)\left(1-\frac{s}{1-\widetilde{s_{n}}}\right)
$$

we have [11]

$$
\begin{aligned}
& \xi(s) \\
& =\xi(0) \prod_{n=1}^{\infty}\left(1-\frac{s}{s_{n}}\right) \\
& =\xi(0) \prod_{n=1}^{\infty}\left(1-\frac{s}{\widetilde{s_{n}}}\right)\left(1-\frac{s}{1-\widetilde{s_{n}}}\right) \\
& =\xi\left(\frac{1}{2}\right) \prod_{n=1}^{\infty}\left[1-\frac{\left(s-\frac{1}{2}\right)^{2}}{\left(\widetilde{s_{n}}-\frac{1}{2}\right)^{2}}\right],
\end{aligned}
$$

where $\xi(0)=1 / 2$, and for $s=1 / 2+i t$ we suggest [11]

$$
\begin{aligned}
& \Xi(t) \\
& =\xi(0) \prod_{n=1}^{\infty}\left(1-\frac{\frac{1}{2}+i t}{s_{n}}\right) \\
& =\xi(0) \prod_{n=1}^{\infty}\left(1-\frac{\frac{1}{2}+i t}{\widetilde{s_{n}}}\right)\left(1-\frac{\frac{1}{2}+i t}{1-\widetilde{s_{n}}}\right), \\
& =\xi\left(\frac{1}{2}\right) \prod_{n=1}^{\infty}\left[1+\frac{t^{2}}{\left(\widetilde{s_{n}}-\frac{1}{2}\right)^{2}}\right] \\
& =\Xi(0) \prod_{n=1}^{\infty}\left[1+\frac{t^{2}}{\left(\widetilde{s_{n}}-\frac{1}{2}\right)^{2}}\right],
\end{aligned}
$$

where $t \in \mathcal{C}$ and

$$
\Xi(0)=\xi\left(\frac{1}{2}\right)>0 .
$$

By using Lemma 5 we have from Eq. (45) that

$$
\Xi(t)=\Xi(0) \prod_{n=1}^{\infty}\left[1+\frac{t^{2}}{\left(\widetilde{s_{n}}-\frac{1}{2}\right)^{2}}\right]=\sum_{h=1}^{\infty} \vartheta(h)(-1)^{h} t^{2 h}
$$


where

$$
\vartheta(h)=\frac{4}{(2 h) !} \int_{1}^{\infty} \frac{d\left(z^{\frac{3}{2}} \psi^{(1)}(x)\right)}{d x} x^{-\frac{1}{4}}\left(\frac{\log x}{2}\right)^{2 h} d x .
$$

By Lemma 6 and the fact $\phi_{n}$ run the Riemann-Siegel zeros, we have

$$
\Xi\left(\phi_{n}\right)=0
$$

such that

$$
\Xi(t)=\Xi(0) \prod_{n=1}^{\infty}\left[1+\frac{\phi_{n}^{2}}{\left(\widetilde{s_{n}}-\frac{1}{2}\right)^{2}}\right]=0
$$

since by Lemma 2, the Turán inequalities

$$
(\vartheta(h))^{2}-\left(\frac{2 h-1}{2 h+1}\right) \vartheta(h-1) \vartheta(h+1)>0
$$

hold for any $h \in \mathcal{N} \cup\{0\}$, and by Lemma 3, the Hardy theorem is valid.

From Eq. (49) we find that

$$
1+\frac{\phi_{n}^{2}}{\left(\widetilde{s_{n}}-\frac{1}{2}\right)^{2}} \neq 0
$$

in which $\Xi(0)>0$.

Thus, from Eq. (51) we have

$$
\widetilde{s_{n}}=\frac{1}{2} \pm i \phi_{n}
$$

and we rewrite Eq. (44) as

$$
\xi(s)=\xi\left(\frac{1}{2}\right) \prod_{n=1}^{\infty}\left[1+\frac{\left(s-\frac{1}{2}\right)^{2}}{\phi_{n}^{2}}\right]
$$

where $\xi\left(\frac{1}{2}\right)>0$ and $t \in \mathcal{C}$.

In view of Lemma 4 we show that there exist $\widetilde{s_{n}}=R e\left(\widetilde{s_{n}}\right)+i \phi_{n}$ such that

$$
\xi\left(\widetilde{s_{n}}\right)=\xi\left(\operatorname{Re}\left(\widetilde{s_{n}}\right)+i \phi_{n}\right)=0 .
$$

Combining Eqs. (53) and (54) we have

$$
1+\frac{\left(\operatorname{Re}\left(\widetilde{s_{n}}\right)+i \phi_{n}-\frac{1}{2}\right)^{2}}{\phi_{n}^{2}}=0,
$$

such that

$$
\left[\left(\operatorname{Re}\left(\widetilde{s_{n}}\right)+i \phi_{n}-\frac{1}{2}\right)-i \phi_{n}\right]\left[\left(\operatorname{Re}\left(s_{n}\right)+i \varphi_{n}-\frac{1}{2}\right)+i \phi_{n}\right]=0 .
$$




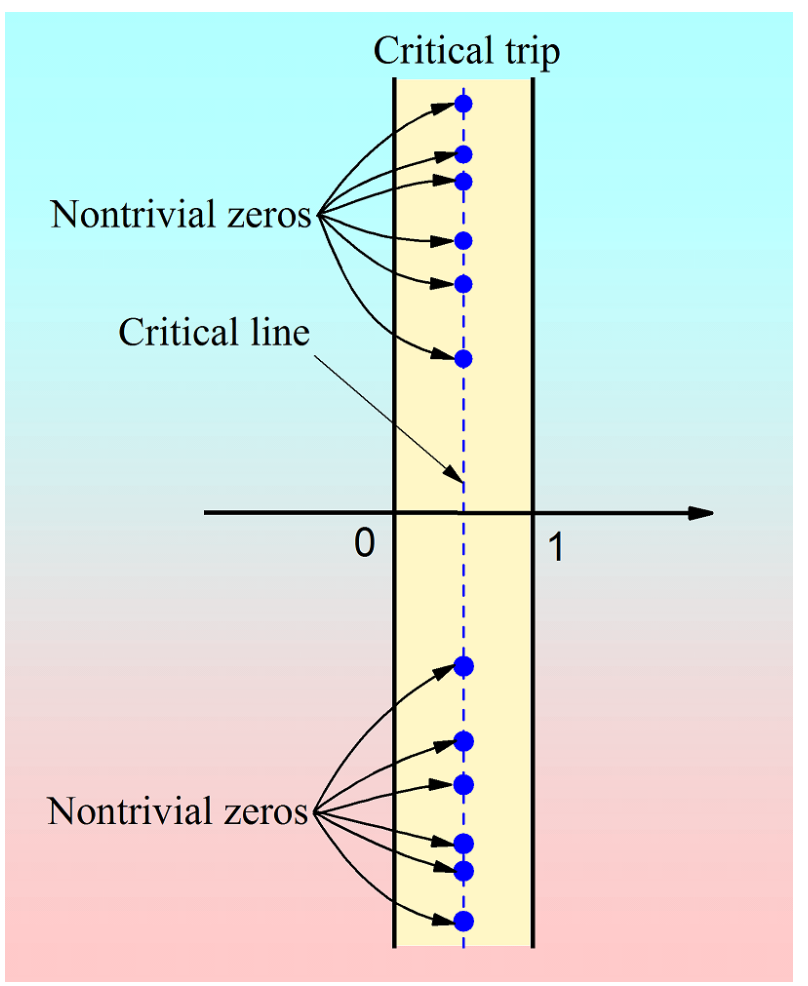

FiguRe 1. All zeros for the entire Riemann zeta function $\xi(s)$ lie on the critical line $\operatorname{Re}(s)=1 / 2$ and in the critical strip $0<\operatorname{Re}(s)<1$.

Because for $\phi_{n} \neq 0$ there exist

$$
\left(R e\left(s_{n}\right)+i \varphi_{n}-\frac{1}{2}\right)+i \phi_{n} \neq 0,
$$

we have

$$
\left(\operatorname{Re}\left(\widetilde{s_{n}}\right)+i \phi_{n}-\frac{1}{2}\right)-i \phi_{n}=0 .
$$

Thus,

$$
\operatorname{Re}\left(\widetilde{s_{n}}\right)=\frac{1}{2},
$$

which is the same as Eq. (52).

This implies that we prove the Heath-Brown statement [7] and that the zeros for $\xi(s)$ are sample [7].

Hence,

$$
\zeta(1 / 2+i t)=\zeta\left(1 / 2+i \phi_{n}\right)=0,
$$


in other worlds that the Riemann theorem follows.

All zeros for the entire Riemann zeta function $\xi(s)$ in the whole complex plane $s$ are demonstrated in Fig. 1.

3.2. The proof of the Lindelöf conjecture. In order to prove the Lindelöf conjecture, from Eq. (38) we have (see [18], p.338)

$$
\Re(T)=\max _{0 \leq t \leq T}|\zeta(1 / 2+i t)| .
$$

Suppose that $\Re(T) \ll T^{\varepsilon}$ for any positive $\varepsilon>0$ and $T \rightarrow \infty$ (see [18], p.338). By Lemma 8 we arrive at

$$
\mu(\sigma)=0
$$

for $\sigma \geq 1 / 2$, and we give

$$
\mu(\sigma)=\frac{1}{2}-\sigma
$$

for $\sigma \leq 1 / 2$.

Since Eq. (60) is valid, we have

$$
\mu\left(\frac{1}{2}\right)=\frac{1}{2}-\frac{1}{2}=0
$$

such that

$$
\Re(T) \ll T^{\varepsilon},
$$

for $T \rightarrow \infty$ and any positive $\varepsilon>0$.

Combining Eqs. (61) and (65) we show that

$$
\zeta(1 / 2+i T) \leq \max _{0 \leq t \leq T}|\zeta(1 / 2+i t)| \ll T^{\varepsilon}
$$

for $T \rightarrow \infty$ and any positive $\varepsilon>0$.

For $T \rightarrow \infty$ and any positive $\varepsilon>0$ we obtain

$$
\zeta(1 / 2+i T) \ll T^{\varepsilon} .
$$

Thus, we finish the proof of the Lindelöf conjecture.

Remark. It has been pointed that the Riemann theorem implies that Lindelöf conjecture $[2,12,22,33]$. 


\section{The moment COnjeCture is true}

In this section we give the proof of the moment conjecture based on the Lindelöf conjecture.

According to Montgomery and Vaughan [18] the alternative representation for the Lindelöf conjecture states that there is

$$
|\zeta(1 / 2+i t)|<\ell t^{\varepsilon},
$$

in which $\ell>0$ is an absolute constant, $t \rightarrow \infty$ and any positive $\varepsilon>0$

By Eq. (68) and $m \in \mathcal{N}$ we have

$$
|\zeta(1 / 2+i t)|^{m}=\left|\zeta^{m}(1 / 2+i t)\right|<\ell^{m} t^{m \varepsilon},
$$

where $\ell>0$ is an absolute constant, $t \rightarrow \infty$ and any positive $\varepsilon>0$

Making use of Eq. (69) there exist for every positive $\varepsilon_{1}=m \varepsilon>0$ and $t \rightarrow \infty$,

$$
\zeta^{m}\left(\frac{1}{2}+i t\right) \ll t^{\varepsilon_{1}}
$$

which is in accordance with the result of Landau [34].

With Eq. (69) we present

$$
\int_{0}^{T}|\zeta(1 / 2+i t)|^{m} d t=\int_{0}^{T}\left|\zeta^{m}(1 / 2+i t)\right| d t<\int_{0}^{T} \ell^{m} t^{m \varepsilon} d t=\frac{\ell^{m}}{m \varepsilon} T^{m \varepsilon+1},
$$

and

$$
\frac{1}{T} \int_{0}^{T}|\zeta(1 / 2+i t)|^{m} d t=\frac{1}{T} \int_{0}^{T}\left|\zeta^{m}(1 / 2+i t)\right| d t<\frac{1}{T} \int_{0}^{T} \ell^{m} t^{m \varepsilon} d t=\frac{\ell^{m}}{m \varepsilon} T^{m \varepsilon+1}
$$

where $\ell>0$ is an absolute constant, $T \rightarrow \infty$ and any positive $\varepsilon>0$.

From Eq. (71) we get

$$
\int_{0}^{T}|\zeta(1 / 2+i t)|^{m} d t \ll t^{\varepsilon_{1}+1}
$$

and from Eq. (72) we have

$$
\frac{1}{T} \int_{0}^{T}|\zeta(1 / 2+i t)|^{m} d t \ll T^{\varepsilon_{1}}
$$

if $T \rightarrow \infty$ and $\varepsilon_{1}=m \varepsilon>0$. 
Similarly, with Eq. (68) we deduce

$$
|\zeta(1 / 2+i t)|^{2 m}=\left|\zeta^{2 m}(1 / 2+i t)\right|<\ell^{2 m} t^{2 m \varepsilon},
$$

in which $\ell>0$ is an absolute constant, $m \in \mathcal{N}, t \rightarrow \infty$ and any positive $\varepsilon>0$.

With use of Eq. (75) there exist

$$
\zeta^{2 m}\left(\frac{1}{2}+i t\right) \ll t^{\varepsilon_{3}}
$$

for every positive $\varepsilon_{3}=2 \varepsilon_{1}>0, m \in \mathcal{N}$ and $t \rightarrow \infty$.

By Eq. (75) we give

$$
\int_{0}^{T}|\zeta(1 / 2+i t)|^{2 m} d t=\int_{0}^{T}\left|\zeta^{2 m}(1 / 2+i t)\right| d t<\int_{0}^{T} \ell^{2 m} t^{2 m \varepsilon} d t=\frac{\ell^{2 m}}{2 m \varepsilon} T^{2 m \varepsilon+1},
$$

and

$$
\frac{1}{T} \int_{0}^{T}|\zeta(1 / 2+i t)|^{2 m} d t=\frac{1}{T} \int_{0}^{T}\left|\zeta^{2 m}(1 / 2+i t)\right| d t<\frac{1}{T} \int_{0}^{T} \ell^{2 m} t^{2 m \varepsilon} d t=\frac{\ell^{2 m}}{2 m \varepsilon} T^{2 m \varepsilon} .
$$

From Eq. (77) we have for any positive $\varepsilon_{4}=2 m \varepsilon>0$ and $T \rightarrow \infty$,

$$
\int_{0}^{T}|\zeta(1 / 2+i t)|^{2 m} d t \ll T^{\varepsilon_{4}+1}
$$

which is in agreement with the result of Laurincikas and Steuding [14].

By virtue of Eq. (78) we present

$$
\frac{1}{T} \int_{0}^{T}|\zeta(1 / 2+i t)|^{2 m} d t \ll T^{\varepsilon_{4}}
$$

which is in accord with the result [15].

Thus, we prove the moment conjecture.

\section{NeW RESUlts on the MOMEnt FOR the RiEmann ZETA FUnCtion}

In this section we propose the new results related to the moment conjecture for the Riemann zeta function. 
Theorem 2. Let $s=\sigma+i t \in \mathcal{C}$ with $\sigma, t \in \mathcal{R}$, and $m \in \mathcal{N}$. Then

$$
\frac{1}{T} \int_{0}^{T}|\zeta(\sigma+i t)|^{m} d t \ll T^{\varepsilon_{1}}
$$

or, alternatively,

$$
\int_{0}^{T}|\zeta(\sigma+i t)|^{m} d t \ll T^{\varepsilon_{1}+1}
$$

where $T \rightarrow \infty$ and any positive $\varepsilon_{1}>0$.

Proof. Since Eq. (5) is valid, there exist an absolute constant $\beta>0, t \rightarrow \infty$ and any positive $\varepsilon>0$ such that

$$
|\zeta(\sigma+i t)|<\beta t^{\varepsilon}
$$

where $\sigma, t \in \mathcal{R}$.

For $m \in \mathrm{N}$ we have from Eq. (83) that

$$
|\zeta(\sigma+i t)|^{m}=\left|\zeta^{m}(\sigma+i t)\right|<\beta^{m} t^{m \varepsilon}=\beta^{m} t^{\varepsilon_{1}},
$$

where $t \rightarrow \infty$.

This implies that

$$
\zeta^{m}(\sigma+i t) \ll t^{\varepsilon_{1}},
$$

in which $\varepsilon_{1}=m \varepsilon>0$.

In view of Eq. (84) we have

$$
\int_{0}^{T}|\zeta(\sigma+i t)|^{m} d t=\int_{0}^{T}\left|\zeta^{m}(\sigma+i t)\right| d t<\int_{0}^{T} \beta^{m} t^{m \varepsilon} d t=\frac{\beta^{m} T^{m \varepsilon+1}}{m \varepsilon}=\frac{\beta^{m}}{\varepsilon_{1}} T^{\varepsilon_{1}+1},
$$

which leads to

$$
\int_{0}^{T}|\zeta(\sigma+i t)|^{m} d t \ll T^{\varepsilon_{1}+1},
$$

where $\varepsilon_{1}=m \varepsilon>0$ and $T \rightarrow \infty$.

From Eq. (86) we get

$\frac{1}{T} \int_{0}^{T}|\zeta(\sigma+i t)|^{m} d t=\frac{1}{T} \int_{0}^{T}\left|\zeta^{m}(\sigma+i t)\right| d t \ll \frac{1}{T} \int_{0}^{T} \beta^{m} t^{m \varepsilon} d t=\frac{1}{T} \frac{\beta^{m} T^{m \varepsilon+1}}{m \varepsilon}=\frac{\beta^{m}}{\varepsilon_{1}} T^{\varepsilon_{1}}$, 
which yields that

$$
\frac{1}{T} \int_{0}^{T}|\zeta(\sigma+i t)|^{m} d t \ll T^{\varepsilon_{1}}
$$

where $\varepsilon_{1}=m \varepsilon>0$ and $T \rightarrow \infty$.

Theorem 3. Let $s=\sigma+i t \in \mathcal{C}$ with $\sigma, t \in \mathcal{R}$, and $m \in \mathcal{N}$. Then

$$
\frac{1}{T} \int_{0}^{T}|\zeta(\sigma+i t)|^{2 m} d t \ll T^{\varepsilon_{3}}
$$

or, alternatively,

$$
\int_{0}^{T}|\zeta(\sigma+i t)|^{2 m} d t \ll T^{\varepsilon_{3}+1}
$$

where $T \rightarrow \infty$.

Proof. For $m \in \mathcal{N}$ we deduce from Eq. (83) that

$$
|\zeta(\sigma+i t)|^{2 m}=\left|\zeta^{2 m}(\sigma+i t)\right|<\beta^{2 m} t^{2 m \varepsilon}=\beta^{2 m} t^{\varepsilon_{3}},
$$

where $t \rightarrow \infty$.

From Eq. (92) we arrive at

$$
\zeta^{2 m}(\sigma+i t) \ll t^{\varepsilon_{3}},
$$

where $\varepsilon_{3}=2 m \varepsilon>0$ and $t \rightarrow \infty$.

With use of Eq. (93) we have

$$
\int_{0}^{T}|\zeta(\sigma+i t)|^{2 m} d t=\int_{0}^{T}\left|\zeta^{2 m}(\sigma+i t)\right| d t<\int_{0}^{T} \beta^{m} t^{2 m \varepsilon} d t=\frac{\beta^{m} T^{2 m \varepsilon+1}}{2 m \varepsilon}=\frac{\beta^{m}}{\varepsilon_{3}} T^{\varepsilon_{3}+1},
$$

which implies that

$$
\int_{0}^{T}|\zeta(\sigma+i t)|^{2 m} d t \ll T^{\varepsilon_{3}+1}
$$

where $\varepsilon_{3}=2 m \varepsilon>0$ and $T \rightarrow \infty$. 
By Eq. (92) we show

(96)

$\frac{1}{T} \int_{0}^{T}|\zeta(\sigma+i t)|^{2 m} d t=\frac{1}{T} \int_{0}^{T}\left|\zeta^{2 m}(\sigma+i t)\right| d t \ll \frac{1}{T} \int_{0}^{T} \beta^{m} t^{2 m \varepsilon} d t=\frac{1}{T} \frac{\beta^{m} T^{2 m \varepsilon+1}}{2 m \varepsilon}=\frac{\beta^{m}}{\varepsilon_{3}} T^{\varepsilon_{3}}$,

which yields that

$$
\frac{1}{T} \int_{0}^{T}|\zeta(\sigma+i t)|^{2 m} d t \ll T^{\varepsilon_{3}}
$$

where $\varepsilon_{3}=2 m \varepsilon>0$ and $T \rightarrow \infty$.

Remark. For $m \in \mathcal{N}$ the following representations for the inequalities are equivalent:

$$
\frac{1}{T} \int_{0}^{T}|\zeta(\sigma+i t)|^{m} d t \ll T^{\varepsilon_{1}}
$$

$$
\int_{0}^{T}|\zeta(\sigma+i t)|^{m} d t \ll T^{\varepsilon_{1}+1}
$$

where $\varepsilon_{1}=m \varepsilon>0$ and $T \rightarrow \infty$, and

$$
\zeta^{m}(\sigma+i t) \ll t^{\varepsilon_{1}},
$$

where $t \rightarrow \infty$.

For $m \in \mathcal{N}$ the following representations for the inequalities are equivalent:

$$
\frac{1}{T} \int_{0}^{T}|\zeta(\sigma+i t)|^{2 m} d t \ll T^{\varepsilon_{3}}
$$

$$
\int_{0}^{T}|\zeta(\sigma+i t)|^{2 m} d t \ll T^{\varepsilon_{3}+1},
$$

where $\varepsilon_{3}=2 m \varepsilon>0$ and $T \rightarrow \infty$, and

$$
\zeta^{m}(\sigma+i t) \ll t^{\varepsilon_{3}}
$$

where $t \rightarrow \infty$.

It is easily seen that the equivalences for Conjecture 1 are true, as shown in $[3,14$, 17]. 
Acknowledgements. This work is supported by the Yue-Qi Scholar of the China University of Mining and Technology (No. 102504180004).

\section{REFERENCES}

[1] Lindelöf, E. (1908). Quelques remarques sur la croissance de la fonction $\zeta(s)$. Bulletin des Sciences Mathématiques, 32, 341-356.

[2] Littlewood, J. E. (1912). Quelques conséquences de l'hypothèse que la fonction $\zeta(s)$ de Riemann n'a pas de zéros dans le demi-plan $R e(s)>1 / 2$, Comptes Rendus de l'Académie des Sciences, $154,263-266$.

[3] Hardy, G. H., Littlewood, J. E. (1923). On Lindelöfs hypothesis concerning the Riemann zeta-function. Proceedings of the Royal Society A: Mathematical, Physical and Engineering Sciences, 103(722), 403-412.

[4] Ingham, A. E. (1964). The distribution of prime numbers. Cambridge University Press, London.

[5] Riemann, G. F. B. (1859). Über die Anzahl der Primzahlen unter einer gegebenen Grösse. Monatsberichte der Deutschen Akademie der Wissenschaften zu Berlin, 2, 671-680.

[6] Titchmarsh, E. C., Heath-Brown, D. R. (1986). The theory of the Riemann zeta-function. Oxford University Press.

[7] Heath-Brown, D. (2005). Prime number theory and the Riemann zeta-function. In F. Mezzadri \& N. Snaith (Eds.), Recent Perspectives in Random Matrix Theory and Number Theory (London Mathematical Society Lecture Note Series, pp. 1-30). Cambridge: Cambridge University Press.

[8] Edwards, H. M. (1974). Riemanns'Zeta Function. Academic press, New York.

[9] Yang, X.-J. (2021). An introduction to hypergeometric, supertrigonometric, and superhyperbolic functions, Academic Press, New York.

[10] Titchmarsh, E. C., Heath-Brown, D. R. (1986). The theory of the Riemann zeta-function. Oxford University Press.

[11] Yang, X.-J. (2021). Analysis of the Riemann zeta function. Reprint, arXiv:1811.02418v10.

[12] Hardy, G. H., Riesz, M. (1915). The general theory of Dirichlet's series. University Press.

[13] Conrey, B. (2015). Riemann's Hypothesis. In L.-Z. Ji, F. Oort, S.-T. Yau (Eds.), The Legacy of Bernhard Riemann After One Hundred and Fifty Years (Advanced Lectures in Mathematics 35, p.107-190). Higher Education Press/International Press.

[14] Laurincikas, A., Steuding, J. (2006). On the Lindelöf hypothesis. Higher Education Press and International Press Beijing-Boston, 17(6), 443-449.

[15] Lifshits, M., Weber, M. (2008). Sampling the Lindelöf Hypothesis with the Cauchy random walk. Proceedings of the London Mathematical Society, 98(1), 241-270.

[16] Conrey, J. B., Ghosh, A. (2006). Remarks on the generalized Lindelöf hypothesis. Functiones et Approximatio Commentarii Mathematici, 36, 71-78.

[17] Fokas, A. S. (2019). A novel approach to the Lindelöf hypothesis. Transactions of Mathematics and Its Applications, 3(1), tnz006.

[18] Montgomery, H. L., Vaughan, R. C. (2006). Multiplicative number theory I: Classical theory. Cambridge university press.

[19] Hadamard, J. (1893). Étude sur les propriétés des fonctions entières et en particulier d'une fonction considérée par Riemann. Journal de Mathématiques Pures et Appliquées, 171-216.

[20] Landau, E. (1909). Handbuch der Lehre von der Verteilung der Primzahlen, Teubner, Leipzig.

[21] Titchmarsh, E. C. (1930). The zeta-function of Riemann, The University Press, Cambridge. 
[22] Patterson, S. J. (1988). An introduction to the theory of the Riemann zeta-function. Cambridge University Press, London.

[23] Csordas, G., Norfolk, T. S., Varga, R. S. (1986). The Riemann hypothesis and the Turán inequalities. Transactions of the American Mathematical Society, 296(2), 521-541.

[24] Csordas, G., Varga, R. S. (1990). Necessary and sufficient conditions and the Riemann hypothesis. Advances in Applied Mathematics, 11(4), 328-357.

[25] Hardy, G. H. (1914). Sur les zéros de la fonction $\zeta(s)$ de Riemann. Comptes Rendus de l'Académie des Sciences de Paris, 158, 1012-1014.

[26] Dimitrov, D. K., Kostov, V. P. (2011). Sharp Turán inequalities via very hyperbolic polynomials. Journal of Mathematical Analysis and Applications, 376(2), 385-392.

[27] Pólya, G., Schur, J. (1914). Über zwei Arten von Faktorenfolgen in der Theorie der algebraischen Gleichungen. Journal für die reine und angewandte Mathematik, 144, 89-113.

[28] Cahen, E. (1894). Sur la fonction $\zeta(s)$ de Riemann et sur des fonctions analogues. Annales scientifiques de l'École Normale Supérieure, 11(3), 75-164.

[29] Titchmarsh, E. C. (1927). A consequence of the Riemann Hypothesis. Journal of the London Mathematical Society, 2(4), 247-254.

[30] Jensen, J. L. (1913). Recherches sur la th'eorie des 'equations. Acta Mathematica, 36(1), 181195.

[31] Pólya, G. (1927). Über die algebraisch-funktionentheoretischen untersuchungen von J. L. W. V. Jensen. Mathematisk-Fysiske Meddelelsev, 17(4), 1-33.

[32] Siegel, C. L. (1932). Über Riemann's Nachlass zur analytischen Zahlentheorie. Quellen und Studien zur Geschichte der Mathematik, Astronomie und Physik, 2 (1932), 45-80 (Reprinted in C. L. Siegel, Gesammelte Abhandlungen, vol. 1, Springer, 1966, pp. 275-310).

[33] Yang, X.-J. (2021). Analysis of the Hamarda function applied in the Riemann, Lindelöf and Hilbert-Pólya conjectures. Reprint, arXiv:1811.02418v8.

[34] Landau, E. (1915). Üeber die Anzahl der Gitterpunkte in Gewissen Bereichen.(Zweite Abhandlung). Nachrichten von der Gesellschaft der Wissenschaften zu Göttingen, MathematischPhysikalische Klasse, 1915, 209-243.

E-mail address: dyangxiaojun@163.com; xjyang@cumt.edu.cn

1 School of Mathematics, China University of Mining and Technology, Xuzhou 221116, CHINA

2 State Key Laboratory for Geomechanics and Deep Underground Engineering, China University of Mining and Technology, Xuzhou 221116, China

3 School of Mechanics and Civil Engineering, China University of Mining and Technology, Xuzhou 221116, China 